\title{
A Postreconstruction Harmonization Method for Multicenter Radiomic Studies in PET
}

\author{
Fanny Orlhac ${ }^{1}$, Sarah Boughdad ${ }^{1,2}$, Cathy Philippe ${ }^{3}$, Hugo Stalla-Bourdillon $^{3}$, Christophe Nioche $^{1}$, Laurence Champion $^{2}$, \\ Michaël Soussan ${ }^{1,4}$, Frédérique Frouin ${ }^{1}$, Vincent Frouin ${ }^{3}$, and Irène Buvat ${ }^{1}$ \\ ${ }^{I}$ Imagerie Moléculaire In Vivo, CEA-SHFJ, INSERM, CNRS, Université Paris-Sud, Université Paris-Saclay, Orsay, France; \\ ${ }^{2}$ Department of Nuclear Medicine, Institut Curie-René Huguenin, Saint-Cloud, France; ${ }^{3}$ NeuroSpin/UNATI, CEA, Université \\ Paris-Saclay, Gif-sur-Yvette, France; and ${ }^{4}$ Department of Nuclear Medicine, AP-HP, Hôpital Avicenne, Bobigny, France
}

Several reports have shown that radiomic features are affected by acquisition and reconstruction parameters, thus hampering multicenter studies. We propose a method that, by removing the center effect while preserving patient-specific effects, standardizes features measured from PET images obtained using different imaging protocols. Methods: Pretreatment ${ }^{18} \mathrm{~F}$-FDG PET images of patients with breast cancer were included. In one nuclear medicine department (department A), 63 patients were scanned on a time-of-flight PET/CT scanner, and 16 lesions were triple-negative (TN). In another nuclear medicine department (department B), 74 patients underwent PET/CT on a different brand of scanner and a different reconstruction protocol, and 15 lesions were $\mathrm{TN}$. The images from department $\mathrm{A}$ were smoothed using a gaussian filter to mimic data from a third department (department A-S). The primary lesion was segmented to obtain a lesion volume of interest (VOI), and a spheric VOI was set in healthy liver tissue. Three SUVs and 6 textural features were computed in all VOls. A harmonization method initially described for genomic data was used to estimate the department effect based on the observed feature values. Feature distributions in each department were compared before and after harmonization. Results: In healthy liver tissue, the distributions significantly differed for 4 of 9 features between departments $A$ and $B$ and for 6 of 9 between departments $\mathrm{A}$ and $\mathrm{A}-\mathrm{S}(P<0.05$, Wilcoxon test). After harmonization, none of the 9 feature distributions significantly differed between 2 departments $(P>0.1)$. The same trend was observed in lesions, with a realignment of feature distributions between the departments after harmonization. Identification of TN lesions was largely enhanced after harmonization when the cutoffs were determined on data from one department and applied to data from the other department. Conclusion: The proposed harmonization method is efficient at removing the multicenter effect for textural features and SUVs. The method is easy to use, retains biologic variations not related to a center effect, and does not require any feature recalculation. Such harmonization allows for multicenter studies and for external validation of radiomic models or cutoffs and should facilitate the use of radiomic models in clinical practice.

Key Words: texture analysis; tumor heterogeneity; PET; radiomics; harmonization

J Nucl Med 2018; 59:1321-1328

DOI: 10.2967/jnumed.117.199935

Received Aug. 2, 2017; revision accepted Dec. 3, 2017.

For correspondence or reprints contact: Fanny Orlhac, IMIV, CEA-SHFJ, INSERM, CNRS, Université Paris-Sud, Université Paris Saclay, 4, Place du Général Leclerc, 91400 Orsay, France.

E-mail: orlhacf@gmail.com

Published online Jan. 4, 2018

COPYRIGHT (C) 2018 by the Society of Nuclear Medicine and Molecular Imaging.
$\mathbf{T}$ he number of publications involving texture analysis or radiomic studies in medical imaging in general, and in PET in particular, is growing rapidly $(1,2)$. Several authors have published promising PET results suggesting that quantification of lesion heterogeneity using radiomic features can predict patient outcome or treatment response (3-6). Yet, in a recent study, Chalkidou et al. (7) pointed out an inappropriate control of type I error in many radiomic studies that involved the calculation of a large number of imaging features in a small number of patients. These authors underlined the need to confirm observations and validate models using independent patient cohorts in multicenter settings. Since the first publication including texture analysis in PET images in 2009 (8), $77 \%$ of the radiomic or texture studies in PET have included fewer than 100 patients (Fig. 1), and only 3 studies involved more than 200 patients (9-11). The difficulty in including a large number of patients lies mostly in the need for a clinically homogeneous cohort with respect to lesion types, stages, treatments, and imaging protocols. Indeed, it has been shown that radiomic features are sensitive to acquisition and reconstruction parameters $(12,13)$, thus hindering the pooling of data acquired using different scanners or protocols. More precisely, radiomic features are sensitive to the reconstruction algorithm, number of iterations or subsets, scan duration per bed position, postreconstruction filter, and voxel size (12-22). This variability of radiomic features implies that a radiomic model established using data from a given PET scanner might not be directly applicable to data from another PET scanner, as recently demonstrated in cervical cancer by Reuzé et al. (23). This is obviously a severe limitation for the dissemination of radiomic models and their transfer to clinical practice.

About 10 years ago, the genomics field faced a similar problem called the batch effect, with batch referring to the settings used to acquire the data, hence being identical to the scanner effect or imaging-protocol effect in radiomics. In genomics, the batch effect is a technical source of variations caused by the handling of samples (e.g., different laboratories, different technicians, different days), potentially masking individual variations, whereas the identification of robust gene signatures to predict disease outcome requires thousands of samples (24). Among the methods developed to deal with the batch effect, ComBat harmonization was described in 2007 (25). This method is now widely used in genomics and has an advantage over other methods in that it provides satisfactory results even for small datasets with a limited number of features (26).

In this context, the purpose of this study was to determine whether the harmonization method initially described for genomic 


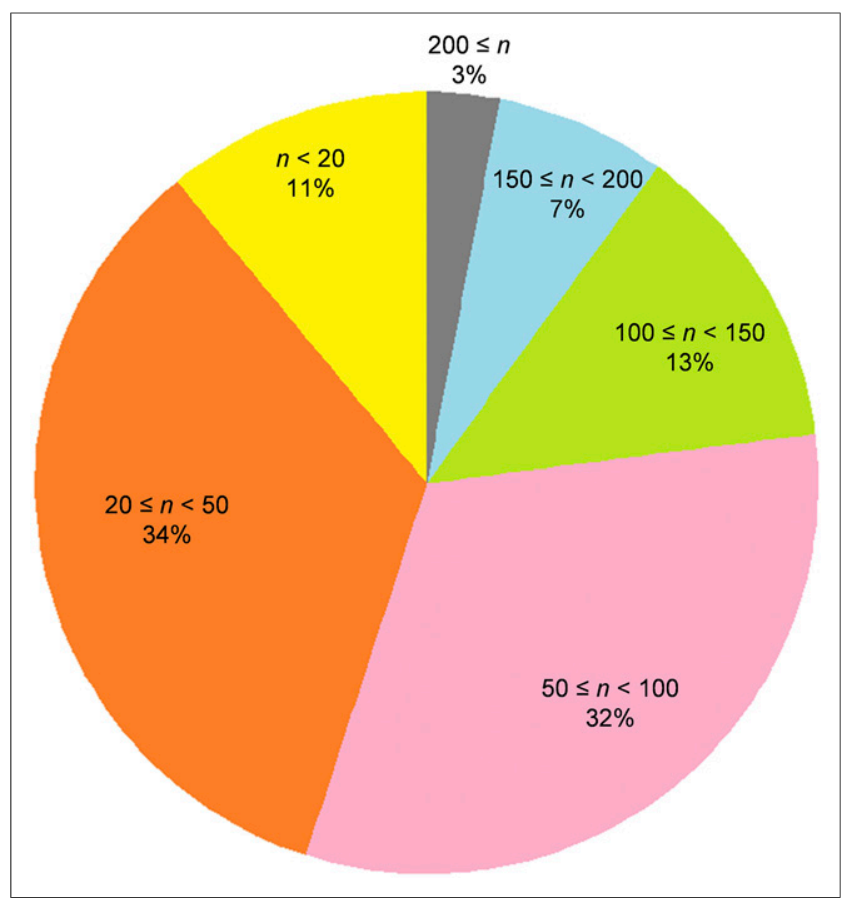

FIGURE 1. Number of patients involved in texture or radiomic studies from PET images since 2009, as found in a PubMed search for "(radiomics OR texture OR textural) AND PET."

data analysis could successfully normalize radiomic features as measured in PET so as to remove the center effect while retaining the pathophysiologic information, in order to facilitate multicenter studies and exportation of a radiomic model to different centers.

\section{MATERIALS AND METHODS}

\section{Patients}

Two groups of patients with nonmetastatic breast cancer, with a total of 137 lesions, were included in this study. The first cohort included 63 patients treated at Avicenne Hospital, Bobigny, France (department A). The second cohort consisted of 74 patients scanned at Institut Curie-René Huguenin Hospital, Saint-Cloud, France (department B). This study was approved by the local institutional review board (Ile-de-France X), and the requirement to obtain informed consent was waived. A core-needle biopsy was performed for all patients to determine the lesion type (27). The characteristics of each patient group are summarized in Table 1.

\section{PET/CT Imaging Protocol}

Each ${ }^{18} \mathrm{~F}$-FDG PET/CT scan was performed before the start of therapy. For each patient, the capillary blood glucose level was less than 8 $\mathrm{mmol} / \mathrm{L}$ at the time of ${ }^{18} \mathrm{~F}$-FDG injection.

In department $\mathrm{A},{ }^{18} \mathrm{~F}-\mathrm{FDG}$ PET/CT images were acquired using a Gemini TF scanner (Philips) at $78 \pm 9$ min (mean \pm SD; range, 59$108 \mathrm{~min})$ after injection of ${ }^{18} \mathrm{~F}-\mathrm{FDG}(3 \mathrm{MBq} / \mathrm{kg})$ at a rate of $1.45 \mathrm{~min}$ per bed position. PET images were reconstructed using a list-mode iterative algorithm (blob ordered-subsets time-of-flight, 2 iterations, 33 subsets). Attenuation was corrected using CT images, and no postreconstruction smoothing was used. The voxel size of the reconstructed PET images was $4 \times 4 \times 4 \mathrm{~mm}$.

In department $\mathrm{B},{ }^{18} \mathrm{~F}-\mathrm{FDG}$ PET/CT images were acquired using a Discovery 690 scanner (GE Healthcare) at $74 \pm 8 \mathrm{~min}$ (range, 55$99 \mathrm{~min})$ after injection of ${ }^{18} \mathrm{~F}-\mathrm{FDG}(3-3.5 \mathrm{MBq} / \mathrm{kg})$ at a rate of $2.5 \mathrm{~min}$ per bed position. PET images were reconstructed using an ordered-subset expectation maximization iterative algorithm (2 iterations, 24 subsets) and gaussian postfiltering $(6 \mathrm{~mm}$ in full width at half maximum). Attenuation was corrected using CT images. The voxel size of the reconstructed PET images was $2.7 \times 2.7 \times 3.3 \mathrm{~mm}$.

Last, we smoothed the PET images from department A using a 3dimensional gaussian filter $(\sigma=4 \mathrm{~mm})$ to mimic a third department (department A-S).

All PET images were converted into SUVs using standardization by patient body weight.

\section{Radiomic Feature Measurements}

For each patient, 2 volumes of interest (VOIs) were delineated. First, we segmented the primary lesion using a fixed threshold of $40 \%$ of its $\mathrm{SUV}_{\max }$. Second, we located a spheric VOI of about $23 \mathrm{~cm}^{3}$ in healthy liver tissue.

For each VOI, 9 features were measured using LIFEx software (www. lifexsoft.org), including $\mathrm{SUV}_{\text {max }}, \mathrm{SUV}_{\text {mean }}$ in the VOI, and $\mathrm{SUV}_{\text {peak }}$ (maximum average SUV in a $1-\mathrm{cm}^{3}$ sphere). For textural feature calculation, voxel intensities were resampled using 64 discrete values between 0 and 20 SUV units, corresponding to an absolute resampling with a bin width of about $0.3 \mathrm{SUV}$ (28). Six textural features previously selected for their robustness with respect to the segmentation method in each texture correlation group (29) were calculated: homogeneity and entropy from the cooccurrence matrix, short-run emphasis and long-run emphasis from the gray-level run length matrix, and high-gray-level zone emphasis and low-gray-level zone emphasis from the gray-level zone length matrix. The method of calculating textural features was previously described in detail (29).

\section{Harmonization Method}

To pool SUV and textural features measured from different PET protocols, we tested a harmonization method, ComBat, previously described for genomic studies by Johnson et al. (25) to correct the batch effect. The harmonization model assumes that the value of each feature $y$ measured in VOI $j$ and scanner $i$ can be written as follows:

$$
y_{i j}=\alpha+X_{i j} \beta+\gamma_{i}+\delta_{i} \varepsilon_{i j}
$$

where $\alpha$ is the average value for feature $y ; X$ is a design matrix for the covariates of interest; $\beta$ is the vector of regression coefficients corresponding to each covariate; $\gamma_{i}$ is the additive effect of scanner $i$ on features, supposed to follow a normal distribution; $\delta_{i}$ is the multiplicative scanner effect, supposed to follow an inverse gamma-distribution; and $\varepsilon_{i j}$ is an error term (normally distributed with a zero mean), as

TABLE 1

Patient Characteristics for Departments A and B

\begin{tabular}{lcc}
\hline Characteristic & A & B \\
\hline Mean age \pm SD $(y)$ & $55 \pm 15$ & $51 \pm 14$ \\
Molecular subtype & & \\
\hline Luminal A & $9(14 \%)$ & $11(15 \%)$ \\
\hline Luminal B & $35(56 \%)$ & $44(59 \%)$ \\
TN & $16(25 \%)$ & $15(20 \%)$ \\
\hline HER2-positive & $3(5 \%)$ & $2(3 \%)$ \\
Unknown & - & $2(3 \%)$ \\
\hline
\end{tabular}

HER2 = human epidermal growth factor receptor 2 .

Data are $n$ followed by percentage, except for age. 


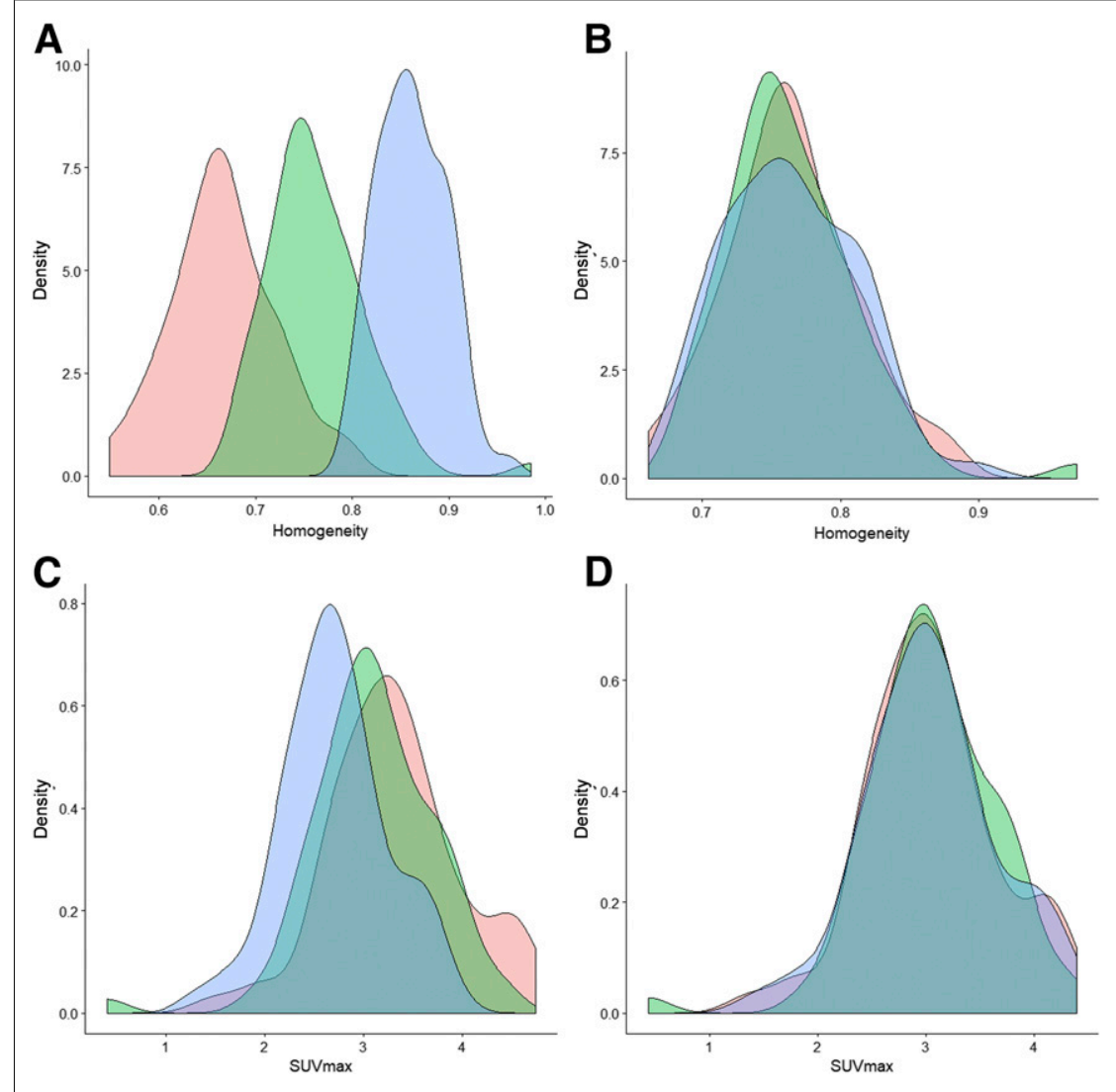

FIGURE 2. Probability density function (\%) of homogeneity (A and B) and $S U V_{\max }(C$ and $D)$ in liver tissue as observed in departments A (pink), B (green), and A-S (blue), before (left) and after (right) harmonization.

explained by Fortin et al. (30). Harmonization consists of estimating $\gamma_{i}$ and $\delta_{i}$ using empiric Bayes estimates (denoted as $\gamma_{i}{ }^{*}$ and $\delta_{i}{ }^{*}$ ) as described previously (25). The same model can be used in its nonparametric form in which no assumptions are made regarding the laws followed by $\gamma_{i}, \delta_{i}$, and $\varepsilon_{i j}$. The normalized value of feature $y$ for VOI $j$ and scanner $i$ is then obtained as follows:

$$
y_{i j}^{\text {ComBat }}=\frac{y_{i j}-\hat{\alpha}-X_{i j} \hat{\beta}-\gamma_{i}^{*}}{\delta_{i}^{*}}+\hat{\alpha}+X_{i j} \hat{\beta} \text {, }
$$

where $\hat{\alpha}$ and $\hat{\beta}$ are estimators of parameters $\alpha$ and $\beta$, respectively. The harmonization determines a transformation for each feature separately, based on the batch (here, department) effect observed on feature values. In the first part of this study, we used harmonization without accounting for any biologic covariate (i.e., $X=0$ ), and in the second part, we used TN status as the covariate of interest.

For each tissue separately (lesion and liver tissues), we applied harmonization on all features using the $\mathrm{R}$ function called ComBat, available at https://github.com/Jfortin1/ComBatHarmonization/, using the non-parametric settings.

\section{Statistical Analysis}

To test the ability of the harmonization method to remove the center effect from the feature values, we plotted the probability density function of all features in the liver VOI as a function of the department, before and after harmonization. We used Wilcoxon tests to determine whether the features differed significantly between departments, with $P$ values of less than 0.05 considered statistically significant.

For the lesion VOI, we displayed the box plots of all features as a function of lesion type-TN or non-TN-for each department separately, before and after harmonization. Wilcoxon tests were used to investigate the impact of harmonization on the features in the TN and non-TN groups in each department.

To study the usefulness of harmonization in multicenter studies, we determined the cutoff for each feature as that maximizing the Youden

TABLE 2

$P$ Values of Wilcoxon Tests Between Feature Values in Departments A and B and in Departments A and A-S Before and After Harmonization

\begin{tabular}{|c|c|c|c|c|}
\hline \multirow[b]{2}{*}{ Feature } & \multicolumn{2}{|c|}{ A vs. $B$} & \multicolumn{2}{|c|}{ A vs. A-S } \\
\hline & Before harmonization & After harmonization & Before harmonization & After harmonization \\
\hline Homogeneity & $<0.0001^{*}$ & 0.7592 & $<0.0001^{*}$ & 0.9300 \\
\hline Entropy & $<0.0001^{*}$ & 0.7828 & $<0.0001^{*}$ & 0.9611 \\
\hline Short-run emphasis & $<0.0001^{*}$ & 0.8930 & $<0.0001^{*}$ & 0.7922 \\
\hline Long-run emphasis & $<0.0001^{*}$ & 0.4708 & $<0.0001^{*}$ & 0.8491 \\
\hline Low-gray-level zone emphasis & 0.5961 & 0.1319 & 0.9397 & 0.9650 \\
\hline High-gray-level zone emphasis & 0.2328 & 0.8100 & $0.0233^{*}$ & 0.8759 \\
\hline$S U V_{\max }$ & 0.0522 & 0.7424 & $<0.0001^{*}$ & 1.0000 \\
\hline $\mathrm{SUV}_{\text {mean }}$ & 0.4042 & 0.8409 & 0.9980 & 1.0000 \\
\hline SUV $_{\text {peak }}$ & 0.3407 & 0.9666 & 0.0614 & 0.9766 \\
\hline
\end{tabular}

${ }^{\star} P<0.05$ 


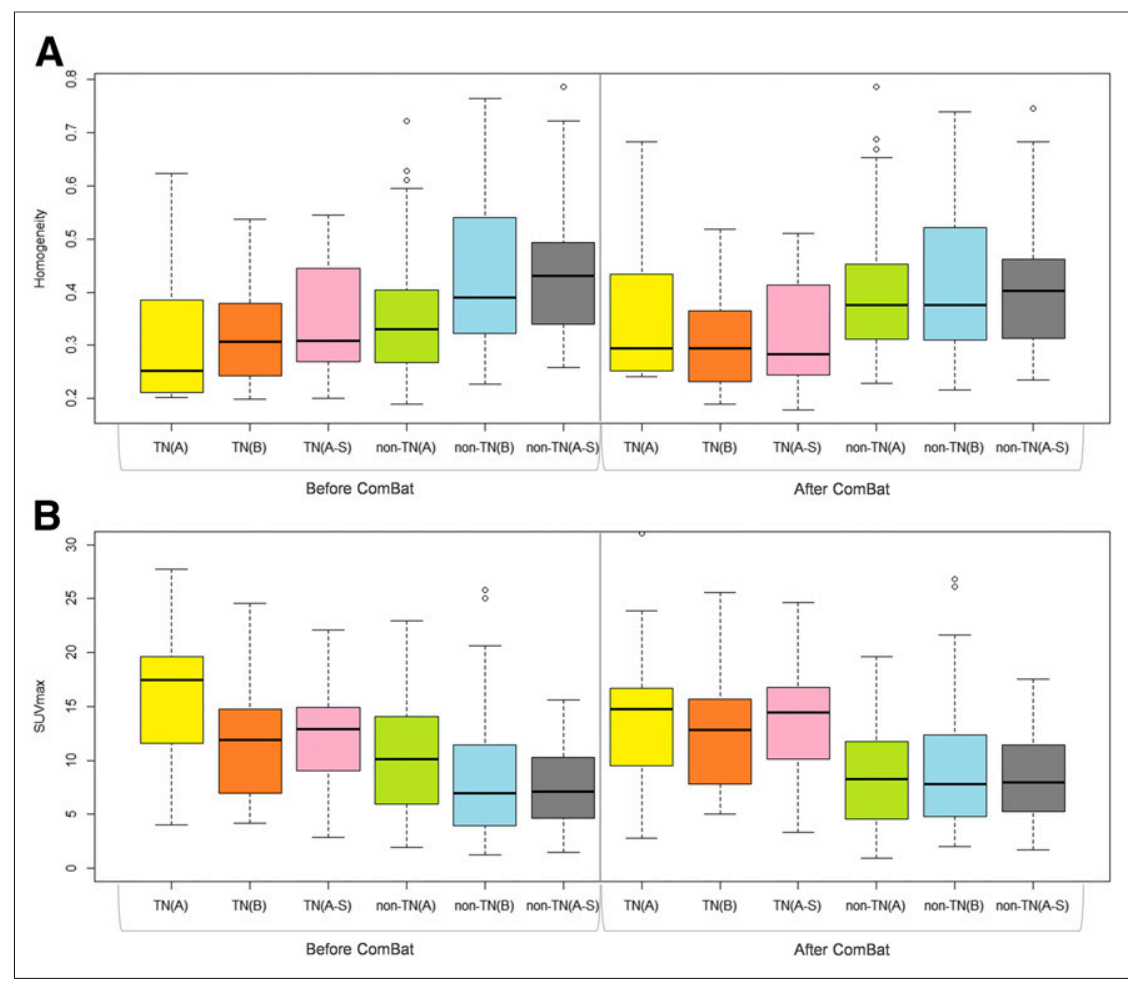

FIGURE 3. Box plots of homogeneity $(\mathrm{A})$ and $\mathrm{SUV}_{\max }(\mathrm{B})$ for TN and non-TN lesions before and after harmonization in 3 departments separately.

index, defined as (sensitivity + specificity -1 ), in separating TN from non-TN groups using data from department $\mathrm{A}$. We then used these cutoffs to determine whether lesions from department B were $\mathrm{TN}$ or non-TN, and we assessed the accuracy of this classification without and with harmonization. The accuracy of the TN classification was also measured using the Youden index.

Finally, we investigated the impact of setting a covariate of interest by removing the $\mathrm{TN}$ lesions from department $\mathrm{A}$ to obtain 2 datasets with different biologic compositions: department A without $\mathrm{TN}$ and department $\mathrm{B}$ including $20 \%$ of $\mathrm{TN}$. We studied how TN lesions in department $\mathrm{B}$ were distinguished from non-TN lesions in department A using Wilcoxon tests before and after harmonization, without and with TN status as a covariate of interest.

\section{RESULTS}

\section{Liver Tissue}

The plots of features in liver tissue show a shift in distribution among the 3 departments (Fig. 2; Supplemental Fig. 1 [supplemental materials are available at http:// jnm.snmjournals.org]). For instance, homogeneity in the liver VOI was lower in department A than in department B or A-S (Fig. 2A). Conversely, $\mathrm{SUV}_{\max }$ was higher in department $\mathrm{A}$ than in $\mathrm{B}$, which was higher than in A-S (Fig. 2C). Homogeneity, entropy, short-run emphasis, and longrun emphasis differed significantly between departments $\mathrm{A}$ and $\mathrm{B}$ and between departments A and A-S $(P<0.05$; Table 2$)$ when the features were not harmonized. Highgray-level zone emphasis and $\mathrm{SUV}_{\max }$ also differed significantly between departments A and A-S.

After harmonization, the distributions overlapped better for all features (Fig. 2; Supplemental Fig. 1), and no feature differed significantly between 2 departments $(P>0.1$; Table 2$)$.

\section{Lesion Tissue}

In our cohorts, 16 of 63 lesions $(25 \%)$ were $\mathrm{TN}$ in departments A and A-S, and 15 of 74 lesions (20\%) were TN in department B (Table 1). The mean lesion volume was $28.1 \pm 39.1 \mathrm{~cm}^{3}$

TABLE 3

$P$ Values of Wilcoxon Tests Between Feature Values in TN and Non-TN Lesions in Departments A and B Before and After Harmonization

\begin{tabular}{|c|c|c|c|c|c|c|c|c|c|c|}
\hline \multirow[b]{2}{*}{ Feature } & \multicolumn{6}{|c|}{ Before harmonization } & \multicolumn{4}{|c|}{ After harmonization } \\
\hline & $\begin{array}{l}\text { TN(A) vs. } \\
\text { non-TN(A) }\end{array}$ & $\begin{array}{l}\text { TN(B) vs. } \\
\text { non-TN(B) }\end{array}$ & $\begin{array}{l}\mathrm{TN}(\mathrm{A}) \text { vs. } \\
\mathrm{TN}(\mathrm{B})\end{array}$ & $\begin{array}{l}\text { Non-TN(A) vs. } \\
\text { non-TN(B) }\end{array}$ & $\begin{array}{c}\mathrm{TN}(\mathrm{A}+\mathrm{B}) \text { vs. } \\
\text { non-TN(A+B) }\end{array}$ & $\begin{array}{c}\text { TN(B) vs. } \\
\text { non-TN(A) }\end{array}$ & $\begin{array}{c}\text { TN(A) vs. } \\
\text { TN(B) }\end{array}$ & $\begin{array}{c}\text { Non-TN(A) vs. } \\
\text { non-TN(B) }\end{array}$ & $\begin{array}{l}\mathrm{TN}(\mathrm{A}+\mathrm{B}) \text { vs. } \\
\text { non-TN(A+B) }\end{array}$ & $\begin{array}{l}\text { TN(B) vs. } \\
\text { non-TN(A) }\end{array}$ \\
\hline Homogeneity & 0.0810 & $0.0078^{*}$ & 0.4232 & $0.0074^{\star}$ & $0.0014^{*}$ & 0.4635 & 0.5986 & 0.8737 & $0.0015^{\star}$ & $0.0093^{*}$ \\
\hline Entropy & $0.0205^{\star}$ & $0.0410^{\star}$ & 0.5196 & 0.3906 & $0.0031^{*}$ & 0.0875 & 0.7405 & 0.9139 & $0.0027^{\star}$ & $0.0254^{\star}$ \\
\hline Short-run emphasis & 0.2175 & $0.0091^{*}$ & 0.2995 & $0.0004^{\star}$ & $0.0063^{*}$ & 0.9481 & 0.1294 & 0.8338 & $0.0062^{\star}$ & $0.0061^{\star}$ \\
\hline Long-run emphasis & 0.2618 & $0.0072^{*}$ & 0.2814 & $0.0004^{*}$ & $0.0072^{*}$ & 0.9352 & $0.0055^{\star}$ & 0.3871 & $0.0162^{*}$ & $0.0004^{*}$ \\
\hline $\begin{array}{l}\text { Low-gray-level } \\
\text { zone emphasis }\end{array}$ & $0.0005^{\star}$ & $0.0119^{*}$ & $0.0405^{\star}$ & $0.0244^{*}$ & $5.69 \mathrm{e}-05^{*}$ & 0.3786 & 0.1102 & 0.3059 & $0.0002^{\star}$ & $0.0003^{*}$ \\
\hline $\begin{array}{l}\text { High-gray-level } \\
\text { zone emphasis }\end{array}$ & $0.0002^{*}$ & $0.0119^{*}$ & $0.0494^{\star}$ & $0.0282^{*}$ & $3.20 \mathrm{e}-05^{*}$ & 0.2886 & 0.2814 & 0.3337 & $2.27 e-05^{\star}$ & $0.0058^{\star}$ \\
\hline SUV $_{\max }$ & $0.0006^{*}$ & $0.0111^{*}$ & 0.0544 & $0.0278^{*}$ & $7.54 \mathrm{e}-05^{\star}$ & 0.4058 & 0.5717 & 0.7943 & $4.47 \mathrm{e}-05^{\star}$ & $0.0072^{*}$ \\
\hline SUV $_{\text {mean }}$ & $0.0003^{*}$ & $0.0139^{\star}$ & $0.0448^{\star}$ & $0.0359^{*}$ & $3.20 \mathrm{e}-05^{*}$ & 0.2394 & 0.4463 & 0.7747 & $3.05 e-05^{\star}$ & $0.0052^{*}$ \\
\hline SUV $_{\text {peak }}$ & $0.0004^{*}$ & $0.0167^{\star}$ & $0.0267^{\star}$ & $0.0306^{\star}$ & $9.75 e-05^{\star}$ & 0.4736 & 0.3581 & 0.7894 & $4.99 \mathrm{e}-05^{\star}$ & $0.0061^{*}$ \\
\hline
\end{tabular}


TABLE 4

Youden Indices for Distinction Between TN and Non-TN Lesions from Department B with Department A-Based Threshold and Department B-Based Threshold

\begin{tabular}{|c|c|c|c|}
\hline \multirow[b]{2}{*}{ Feature } & \multicolumn{2}{|c|}{ Thresh. A } & \multirow[b]{2}{*}{ Thresh. B } \\
\hline & Before harmonization & After harmonization & \\
\hline Homogeneity & 0.23 & 0.28 & 0.36 \\
\hline Entropy & 0.21 & 0.20 & 0.39 \\
\hline Short-run emphasis & 0.12 & 0.35 & 0.38 \\
\hline Long-run emphasis & 0.08 & 0.28 & 0.41 \\
\hline Low-gray-level zone emphasis & 0.07 & 0.33 & 0.39 \\
\hline High-gray-level zone emphasis & 0.16 & 0.21 & 0.39 \\
\hline$S U V_{\text {mean }}$ & 0.15 & 0.30 & 0.37 \\
\hline$S_{\text {SUax }}$ & 0.05 & 0.25 & 0.32 \\
\hline SUV $_{\text {peak }}$ & 0.05 & 0.36 & 0.37 \\
\hline
\end{tabular}

(range: $\left.4.8-229.1 \mathrm{~cm}^{3}\right)$ in departments $\mathrm{A}$ and A-S and $12.3 \pm 13.0$ $\mathrm{cm}^{3}$ (range: $\left.2.0-77.3 \mathrm{~cm}^{3}\right)$ in department $\mathrm{B}(P<0.05)$.

Figure 3 and Supplemental Figure 2 show that, in each department separately, uptake heterogeneity was higher in $\mathrm{TN}$ than non-TN lesions, with lower values for homogeneity, long-run emphasis, and low-gray-level zone emphasis and higher values for entropy, short-run emphasis, high-gray-level zone emphasis, and SUV.

Before harmonization, we observed a shift between features for the lesion VOI among the 3 departments (Supplemental Fig. 2) with, for example, a lower homogeneity and a higher $\mathrm{SUV}_{\max }$ in department A than in department B or A-S (Fig. 3; Supplemental

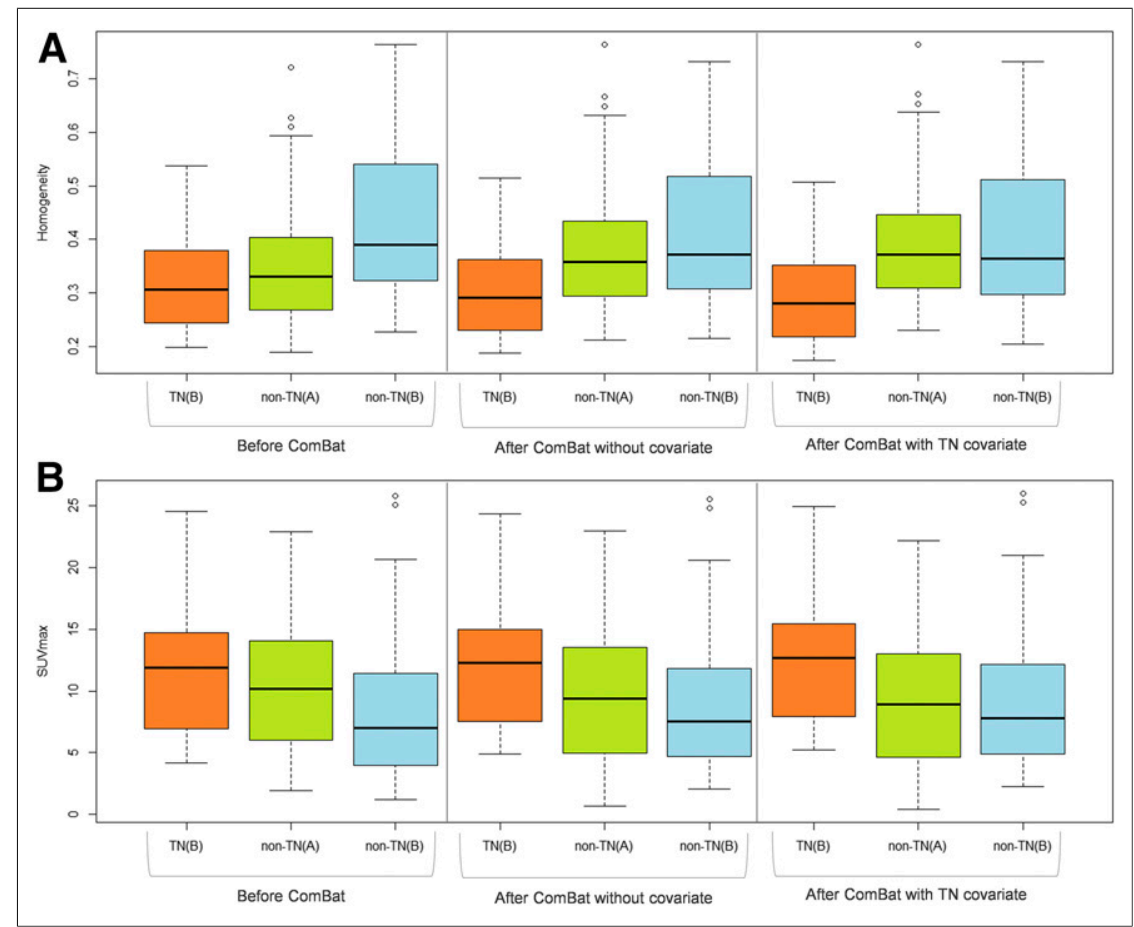

FIGURE 4. Box plots of homogeneity $(A)$ and $S U V_{\max }(B)$ for $T N$ and non-TN lesions before and after harmonization without and with TN status as covariate, for departments $A$ and $B$ separately when all TN are removed from department $A$ to determine transformations.
Fig. 3). Table 3 shows that no feature could distinguish between non-TN lesions from department A and TN lesions from department B $(P>0.08)$. Five of 9 features did not significantly differ between non-TN lesions from department $\mathrm{A}$ and $\mathrm{TN}$ lesions from department A-S (Supplemental Table 1). All features differed significantly between the 2 types of lesions in each center separately, excepting short-run emphasis and long-run emphasis in departments $\mathrm{A}$ and $\mathrm{A}-\mathrm{S}$ and homogeneity in department A.

Figure 3 and Supplemental Figure 2 show that, after harmonization, there was a realignment of features among the 3 departments for TN and non-TN lesions. Table 3 shows that before harmonization, there was a significant difference between departments A and $\mathrm{B}$ for 4 of 9 features in TN lesions and 8 of 9 features in non-TN lesions. Supplemental Table 1 shows that before harmonization, 7 of 9 features in non-TN lesions differed significantly between departments A and A-S. After harmonization, the only feature (for either lesion type) that differed significantly between either A and B or A and $\mathrm{A}-\mathrm{S}$ was long-run emphasis in $\mathrm{TN}$ lesions between A and B (Table 3; Supplemental Table 1; Supplemental Fig. 3). The $P$ values for distinguishing between nonTN lesions in department A and TN lesions in department B or A-S were always lower with harmonization than without (Table 3; Supplemental Table 1).

To mimic a multicenter study, we determined for each feature a cutoff to distinguish between TN and non-TN lesions based on data from department A and then applied these cutoffs to data from department B. Table 4 shows that, before harmonization, all Youden indices were between 0.05 and 0.23 , reflecting poor to moderate distinction between $\mathrm{TN}$ and nonTN lesions. After harmonization, the Youden indices for 8 of 9 features increased to between 0.20 and 0.36 , significantly higher than before harmonization $(P=0.008)$. 
These Youden indices after harmonization were close to those obtained when the cutoff was directly based on data from department B (Table 4). For instance, with a department A-based cutoff, the Youden index for $\mathrm{SUV}_{\text {peak }}$ for department B was 0.05 before harmonization and increased to 0.36 after harmonization-similar to that $(0.37)$ obtained with a department B-based cutoff.

\section{Setting a Covariate of Interest}

When we removed the TN lesions from department A and applied harmonization for departments $\mathrm{A}$ and $\mathrm{B}$, the $P$ values for distinguishing between TN lesions in department B and non-TN lesions in department A decreased for 8 of 9 features compared with $P$ values obtained before harmonization (Fig. 4; Supplemental Fig. 4; Supplemental Table 2). The $P$ values further decreased when the TN status was set as a covariate of interest in the harmonization; all features were then statistically significant for distinguishing between TN lesions in department B and non-TN lesions in department A (Supplemental Table 2).

\section{DISCUSSION}

In this study, we demonstrated that it is possible to pool radiomic features and SUV measurements from different PET imaging protocols by applying a harmonization method, ComBat, initially used in genomics. The efficiency of the method was illustrated using measurements of healthy liver tissue and breast lesions on ${ }^{18} \mathrm{~F}-\mathrm{FDG}$ PET images acquired in 2 different departments and with significantly different spatial resolutions (i.e., the same images acquired with and without gaussian smoothing of $9.4 \mathrm{~mm}$ in full width at half maximum). In addition, we found that with this method, a lesion-subtype-distinguishing cutoff established using data from one PET scanner is applicable to data from another PET scanner.

Among the methods developed to deal with the batch effect, the ComBat harmonization has already been used to normalize histopathologic images for cancer diagnosis (31) and cortical-thickness measurements from MR images (32). The method has several advantages. It is easily accessible, practical (thanks to an $\mathrm{R}$ function available for free), and fast. It is a department-specific harmonization that is based only on patient data acquired in the different departments, and it does not require any phantom experiment, making it suitable for retrospective data analysis. An additional advantage is that it applies directly to the radiomic features and not to the PET images from which those features are calculated. Therefore, it does not reduce the quality of an image set to match the lower quality of other sets. Without covariates of interest, affine transformations are used to harmonize the features. The transformations are different for each feature, each VOI type, and each department, so that the transformed data lie in a common space in which the department effects have been removed or at least reduced. Because the transformations are estimated and applied to the measured data themselves, the transformed data can be pooled afterward without the need for learning sets. The only constraint is that data from the different departments must be available so that the transformations can be identified. In the context of radiomic modeling, this implies that when a published predictive radiomic model is based on data from a certain department, that department must make available the radiomic feature values used to establish the model, as well as the model equations and coefficients, if the model is to be applicable to data from a different department. 
Despite continuous and commendable efforts by the international community and societies to produce guidelines for harmonized imaging procedures (33-35), the acquisition and reconstruction protocols for PET images are not yet standardized. The method we propose offers a solution to performing multicenter studies even when data have been acquired under different conditions. ComBat harmonization is usable not only for radiomic textural features but also for SUV measurements. In our data, we observed that $\mathrm{SUV}_{\max }$ distributions in the liver differed between departments $\mathrm{A}$ and $\mathrm{B}$ $(P=0.05)$ before harmonization but overlapped better after harmonization (Table 2; Fig. 2). Similarly, all SUV $P$ values were greater than 0.2 between non-TN lesions from department $\mathrm{A}$ and TN lesions from department B (Table 3), meaning that SUV measurements failed to distinguish TN from non-TN lesions. After harmonization, SUV $P$ values were less than 0.007 between nonTN lesions from department A and TN lesions from department B, close to the $P$ values observed when distinguishing between TN and non-TN lesions in department $\mathrm{A}(P \leq 0.006)$ and in department $\mathrm{B}(P \leq 0.02)$. This harmonization method is therefore useful for SUVs in a multicenter investigation or for retrospective analysis of PET images acquired on different scanners at the same institution or on one scanner but with different acquisition and reconstruction parameters. Supplemental Figure 3 demonstrates how harmonization greatly reduced the differences in SUVs or feature values between departments A-S and A, although some differences remained for individual lesions. Residual differences after harmonization are also due to the strong smoothing in A-S data, which induces some information loss that obviously cannot be recovered using harmonization.

In each of the 3 PET image-based radiomic studies that included more than 200 patients (Fig. 1) -one to evaluate somatic mutations (9), another to predict prognosis in non-small cell lung cancer (10), and a third to predict treatment response in esophageal cancer (11) - the PET images came from different PET scanners but the scanner effect was not explicitly accounted for. Many studies have reported the impact of acquisition and reconstruction parameters on radiomic features. Ignoring the scanner effect when pooling data from different centers can affect the results in two ways: either making the results more significant than they are or hiding significant differences (Fig. 5).

Although entropy was the most robust feature in previous publications (Supplemental Table 4 of Orlhac et al. (22)), we observed a shift in entropy in liver tissue among the 3 departments (Supplemental Fig. 1A), with higher entropy in department A than in department B or A-S $(P<0.0001$; Table 2$)$. The shift was no longer present after harmonization $(P>0.7)$, suggesting that even when a feature is robust with respect to different imaging protocols, a scanner effect can still be present and require compensation in multicenter studies.

If covariates of interest are properly set (Eq. 1), ComBat harmonization may be used even if the patient groups have different characteristics, as explained by Fortin et al. (32). ComBat harmonization therefore removes the center effect without altering the biologic information conveyed by the radiomic features. When patient characteristics are very different between departments, the ComBat harmonization procedure should define any covariates that are to be protected - that is, are not to enter the harmonization process. For example, when removing the TN lesions from department A, we observed better discrimination between TN lesions from department B and non-TN lesions from department A (Supplemental Table 2) if TN status was defined as a covariate (using the $X$ design matrix (Eq. 1)) than when no covariate was defined. Yet, with ComBat harmonization being a data-driven technique, it is preferable to use clinically and biologically similar datasets whenever possible.

ComBat has been described in a parametric version, with assumptions about the statistical distribution of the model parameters, and in a non-parametric version (25). Because our data did not closely fulfil these assumptions, we used the nonparametric version instead.

Differences between images from different PET systems could also be due to calibration differences. We performed a test (data not shown) to ensure that the image differences removed by ComBat harmonization could not have been sufficiently removed by simple corrections involving a rescaling or offset factor estimated from healthy liver.

A limitation of our study was the small number of patients in each department, although the number was consistent with most PET radiomic studies (Fig. 1). Studies of other imaging protocols and other cancer types are needed to more extensively validate the radiomic use of ComBat harmonization. The minimum number of patients required per department should also be further explored, especially in the nonparametric setting. ComBat has been specifically designed to be robust to small samples $(25,32)$ and has been used in genomics with as few as 25 samples in each batch (25).

\section{CONCLUSION}

Using ComBat harmonization, a procedure initially described for genomic analysis, we showed that radiomic feature values and SUVs from images acquired in different departments or under different conditions could be pooled for further analysis. Harmonization preserved the individual variations in healthy liver tissue and breast lesions while removing the imaging-protocol effect. This method is easily available and does not require any feature recalculation since it applies directly to the radiomic feature values as opposed to the images. The ComBat harmonization approach appears to be a promising way to build radiomic models using data pooled from different departments. This harmonization method is also useful to apply a radiomic model derived from images acquired in one department to images acquired in another department.

\section{DISCLOSURE}

This work was supported by the "Lidex-PIM" project funded by the IDEX Paris-Saclay, ANR-11-IDEX-0003-02. No other potential conflict of interest relevant to this article was reported.

\section{REFERENCES}

1. Alic L, Niessen WJ, Veenland JF. Quantification of heterogeneity as a biomarker in tumor imaging: a systematic review. PLoS One. 2014;9:e110300.

2. Gillies RJ, Kinahan PE, Hricak H. Radiomics: images are more than pictures, they are data. Radiology. 2016;278:563-577.

3. Ha S, Park S, Bang J-I, Kim E-K, Lee H-Y. Metabolic radiomics for pretreatment ${ }^{18}$ F-FDG PET/CT to characterize locally advanced breast cancer: histopathologic characteristics, response to neoadjuvant chemotherapy, and prognosis. Sci Rep. 2017;7:1556.

4. Ben Bouallègue F, Al Tabaa Y, Kafrouni M, Cartron G, Vauchot F, MarianoGoulart D. Association between textural and morphological tumor indices on baseline PET-CT and early metabolic response on interim PET-CT in bulky malignant lymphomas. Med Phys. 2017;44:4608-4619.

5. Desbordes P, Ruan S, Modzelewski R, et al. Predictive value of initial FDG-PET features for treatment response and survival in esophageal cancer patients treated with chemo-radiation therapy using a random forest classifier. PLoS One. 2017;12:e173208. 
6. Beukinga RJ, Hulshoff JB, van Dijk LV, et al. Predicting response to neoadjuvant chemoradiotherapy in esophageal cancer with textural features derived from pretreatment ${ }^{18}$ F-FDG PET/CT imaging. J Nucl Med. 2017;58:723-729.

7. Chalkidou A, O'Doherty MJ, Marsden PK. False discovery rates in PET and CT studies with texture features: a systematic review. PLoS One. 2015;10: e0124165.

8. El Naqa I, Grigsby P, Apte A, et al. Exploring feature-based approaches in PET images for predicting cancer treatment outcomes. Pattern Recognit. 2009;42:11621171.

9. Yip SSF, Kim J, Coroller TP, et al. Associations between somatic mutations and metabolic imaging phenotypes in non-small cell lung cancer. J Nucl Med. 2017; 58:569-576.

10. Ohri N, Duan F, Snyder BS, et al. Pretreatment ${ }^{18}$ F-FDG PET textural features in locally advanced non-small cell lung cancer: secondary analysis of ACRIN 6668/ RTOG 0235. J Nucl Med. 2016;57:842-848.

11. van Rossum PSN, Fried DV, Zhang L, et al. The incremental value of subjective and quantitative assessment of ${ }^{18} \mathrm{~F}$-FDG PET for the prediction of pathologic complete response to preoperative chemoradiotherapy in esophageal cancer. J Nucl Med. 2016;57:691-700.

12. Galavis PE, Hollensen C, Jallow N, Paliwal B, Jeraj R. Variability of textural features in FDG PET images due to different acquisition modes and reconstruction parameters. Acta Oncol. 2010;49:1012-1016.

13. Yan J, Chu-Shern JL, Loi HY, et al. Impact of image reconstruction settings on texture features in ${ }^{18}$ F-FDG PET. J Nucl Med. 2015;56:1667-1673.

14. Doumou G, Siddique M, Tsoumpas C, Goh V, Cook GJ. The precision of textural analysis in ${ }^{18}$ F-FDG-PET scans of oesophageal cancer. Eur Radiol. 2015;25:28052812.

15. Nyflot MJ, Yang F, Byrd D, Bowen SR, Sandison GA, Kinahan PE. Quantitative radiomics: impact of stochastic effects on textural feature analysis implies the need for standards. J Med Imaging (Bellingham). 2015;2:041002.

16. van Velden FHP, Kramer GM, Frings V, et al. Repeatability of radiomic features in non-small-cell lung cancer $\left[{ }^{18} \mathrm{~F}\right] \mathrm{FDG}-\mathrm{PET} / \mathrm{CT}$ studies: impact of reconstruction and delineation. Mol Imaging Biol. 2016;18:788-795.

17. Bailly C, Bodet-Milin C, Couespel S, et al. Revisiting the robustness of PETbased textural features in the context of multi-centric trials. PLoS One. 2016;11: e0159984.

18. Forgacs A, Pall Jonsson H, Dahlbom M, et al. A study on the basic criteria for selecting heterogeneity parameters of F18-FDG PET images. PLoS One. 2016; 11:e0164113.

19. Lasnon C, Majdoub M, Lavigne B, et al. ${ }^{18}$ F-FDG PET/CT heterogeneity quantification through textural features in the era of harmonisation programs: a focus on lung cancer. Eur J Nucl Med Mol Imaging. 2016;43:2324-2335.
20. Orlhac F, Thézé B, Soussan M, Boisgard R, Buvat I. Multi-scale texture analysis: from ${ }^{18}$ F-FDG PET images to pathological slides. J Nucl Med. 2016;57:18231828.

21. Shiri I, Rahmim A, Ghaffarian P, Geramifar P, Abdollahi H, Bitarafan-Rajabi A. The impact of image reconstruction settings on ${ }^{18} \mathrm{~F}$-FDG PET radiomic features: multi-scanner phantom and patient studies. Eur Radiol. 2017;27:4498-4509.

22. Orlhac F, Nioche C, Soussan M, Buvat I. Understanding changes in tumor texture indices in PET: a comparison between visual assessment and index values in simulated and patient data. J Nucl Med. 2017;58:387-392.

23. Reuzé S, Orlhac F, Chargari C, et al. Prediction of cervical cancer recurrence using textural features extracted from ${ }^{18} \mathrm{~F}-\mathrm{FDG}$ PET images acquired with different scanners. Oncotarget. 2017;8:43169-43179.

24. Lazar C, Meganck S, Taminau J, et al. Batch effect removal methods for microarray gene expression data integration: a survey. Brief Bioinform. 2013;14:469-490.

25. Johnson WE, Li C, Rabinovic A. Adjusting batch effects in microarray expression data using empirical Bayes methods. Biostatistics. 2007;8:118-127.

26. Goh WWB, Wang W, Wong L. Why batch effects matter in omics data, and how to avoid them. Trends Biotechnol. 2017;35:498-507.

27. Soussan M, Orlhac F, Boubaya M, et al. Relationship between tumor heterogeneity measured on FDG-PET/CT and pathological prognostic factors in invasive breast cancer. PLoS One. 2014;9:e94017.

28. Orlhac F, Soussan M, Chouahnia K, Martinod E, Buvat I. ${ }^{18}$ F-FDG PET-derived textural indices reflect tissue-specific uptake pattern in non-small cell lung cancer. PLoS One. 2015;10:e0145063.

29. Orlhac F, Soussan M, Maisonobe J-A, Garcia CA, Vanderlinden B, Buvat I. Tumor texture analysis in ${ }^{18} \mathrm{~F}-\mathrm{FDG}$ PET: relationships between texture parameters, histogram indices, standardized uptake values, metabolic volumes, and total lesion glycolysis. J Nucl Med. 2014;55:414-422.

30. Fortin J-P, Parker D, Tunc B, et al. Harmonization of multi-site diffusion tensor imaging data. Neuroimage. 2017;161:149-170.

31. Kothari S, Phan JH, Stokes TH, Osunkoya AO, Young AN, Wang MD. Removing batch effects from histopathological images for enhanced cancer diagnosis. IEEE J Biomed Health Inform. 2014;18:765-772.

32. Fortin J-P, Cullen N, Sheline YI, et al. Harmonization of cortical thickness measurements across scanners and sites. Neuroimage. 2017;167:104-120.

33. Delbeke D, Coleman RE, Guiberteau MJ, et al. Procedure guideline for tumor imaging with ${ }^{18}$ F-FDG PET/CT 1.0. J Nucl Med. 2006;47:885-895.

34. Boellaard R, Delgado-Bolton R, Oyen WJG, et al. FDG PET/CT: EANM procedure guidelines for tumour imaging: version 2.0. Eur J Nucl Med Mol Imaging. 2015;42:328-354.

35. Clarke LP, Nordstrom RJ, Zhang $\mathrm{H}$, et al. The quantitative imaging network: NCI's historical perspective and planned goals. Transl Oncol. 2014;7:1-4. 